\title{
ORDEN DE ADQUISICIÓN EN ESPAÑOL COMO LENGUA EXTRANJERA: MODALIDAD SUBJUNTIVA *
}

\author{
Carlos Contreras Aedo** \\ Anita Ferreira Cabrera***
}

\section{Resumen}

En este artículo se presentan resultados cualitativos de un estudio exploratorio del orden de adquisición del español como lengua extranjera en estudiantes anglo, franco y germano parlantes. Se incluyen indicaciones de Collentine (2010) y resultados de Montrul (2008) relacionados con la adquisición del pretérito indefinido/imperfecto del modo indicativo y el presente de subjuntivo en ELE. Los resultados obtenidos revelan un orden de adquisición del pretérito indefinido/imperfecto previo a la adquisición del presente de subjuntivo, transferencia desde la L1 y una distribución de los errores en oraciones subordinadas que es diferente en las tres lenguas maternas estudiadas.

Palabras clave: ASL, ELE, L1, secuencia de adquisición, subjuntivo, transferencia.

\section{L2 SPANISH ACQUISITION ORDER: SUBJUNTIVE MOOD}

\begin{abstract}
This article presents qualitative results of an exploratory study of the order of acquisition of Spanish as a foreign language in native speakers of English, French and German. Indications given by Collentine (2010) and Montrul (2008) related to the acquisition of L2 Spanish preterit/imperfect and present subjunctive are included. The results show an order of acquisition of the preterit/imperfect prior to the acquisition of the present subjunctive; in addition, they show transfer from L1 and an error distribution pattern in subordinate clauses which is different in the three native languages studied.
\end{abstract}

Key words: acquisition sequence, L1, L2 Spanish, SLA, transfer, subjunctive

Recibido: 20-01-2012

Aceptado: 22-01-2013

* Este artículo es parte de la tesis doctoral en Lingüística Aplicada, Retroalimentación correctiva implícita en español como lengua extranjera, Universidad de Concepción.

** Chileno, Magíster en Lingüística Aplicada, Universidad de Concepción, Concepción, Chile carlcont@udec.cl

*** Chilena, Doctora en Inteligencia Artificial, Universidad de Concepción, Concepción, Chile, aferreir@udec.cl 


\section{Introducción}

En materia de adquisición de segundas lenguas (ASL), el marco común europeo de referencia para las lenguas (MCER) es un estándar propuesto en un congreso internacional en Suiza en 1991 que ha sido desarrollado por el Consejo de Europa como patrón internacional para medir el nivel de comprensión y expresión orales y escritas en una lengua.

El MCER elabora una distinción inicial en tres bloques amplios, cada uno de los cuales se ramifica en otros dos niveles más restrictivos. Establece así una serie de tres bloques y seis niveles para la comparación, el aprendizaje, la enseñanza y evaluación de las lenguas, y la homologación de los distintos títulos emitidos por entidades certificadas.

Para el caso del español como lengua extranjera (ELE), a partir de los descriptores del Nivel A2 en el bloque de usuario básico, el MCER indica:

Se adquiere cuando el estudiante es capaz de comprender frases y expresiones de uso frecuente relacionadas con áreas de experiencia que le son especialmente relevantes (información básica sobre sí mismo y su familia, compras, lugares de interés, ocupaciones, etc.); cuando sabe comunicarse a la hora de llevar a cabo tareas simples y cotidianas que no requieran más que intercambios sencillos y directos de información sobre cuestiones que le son conocidas o habituales y cuando sabe describir en términos sencillos aspectos de su pasado y su entorno así como cuestiones relacionadas con sus necesidades inmediatas. (26).

Los descriptores del nivel siguiente, B1 del bloque de usuario independiente, indican:

Se adquiere cuando el estudiante es capaz de comprender los puntos principales de textos claros y en lengua estándar si tratan sobre cuestiones que le son conocidas, ya sea en situaciones de trabajo, de estudio o de ocio; cuando sabe desenvolverse en la mayor parte de las situaciones que pueden surgir durante un viaje por zonas donde se utiliza la lengua; cuando es capaz de producir textos sencillos y coherentes sobre temas que le son familiares o en los que tiene un interés personal y cuando puede describir experiencias, acontecimientos, deseos y aspiraciones, así como justificar brevemente sus opiniones o explicar sus planes. (26) 
Específicamente para las habilidades de lectura, producción oral y escrita, los descriptores señalan:

\section{Comprensión de lectura B1:}

Comprendo textos redactados en una lengua de uso habitual y cotidiano o relacionada con el trabajo. Comprendo la descripción de acontecimientos, sentimientos y deseos en cartas personales. (30)

\section{Expresión oral B1:}

Sé enlazar frases de forma sencilla con el fin de describir experiencias y hechos, mis sueños, esperanzas y ambiciones. Puedo explicar y justificar brevemente mis opiniones y proyectos. Sé narrar una historia o relato, la trama de un libro o película y puedo describir mis reacciones. (30).

\section{Expresión escrita B1:}

Soy capaz de escribir textos sencillos y bien enlazados sobre temas que me son conocidos o de interés personal. $\underline{\text { Puedo }}$ escribir cartas personales que describen experiencias e impresiones. (30).

Ateniéndonos a estos descriptores, observamos que la habilidad de describir experiencias en pasado, narrar una historia o relato, o bien, la trama de un libro, etc., implica el conocimiento de los aspectos perfectivo e imperfectivo expresados por los pretéritos indefinido e imperfecto, respectivamente, del modo indicativo en español. Por su parte, la capacidad de expresar sueños, esperanzas, ambiciones, impresiones y reacciones personales requiere el conocimiento de la representación inflexional de la modalidad subjuntiva que señala una ausencia de compromiso con el valor de verdad de un enunciado (Palmer 2001), lo cual entraña participación de los niveles semánticos y pragmáticos de la lengua, habitualmente en contextos de subordinación.

Es así que los pretéritos indefinido e imperfecto del modo indicativo y el presente del modo subjuntivo constituyen dos de los mayores desafíos para estudiantes de ELE incluso en un nivel avanzado. De los descriptores del MCER inferimos que las estructuras involucradas en tales desafíos coinciden en el nivel B1: “describir experiencias y hechos, mis sueños, 
esperanzas y ambiciones; narrar una historia o relato, la trama de un libro o película y puedo describir mis reacciones". Asimismo, dado que el MCER no proporciona los contenidos gramaticales incluidos en cada nivel, inferimos a partir de la descripción del nivel A2 que la adquisición y el uso de los pretéritos indefinido e imperfecto (" términos sencillos aspectos de su pasado") se esperan en la etapa inmediatamente anterior a aquella en la que se esperan la adquisición y el uso del presente de subjuntivo.

Diversos autores afirman que el modo subjuntivo, el tema principal en este estudio, tiene una frecuencia de uso relativamente baja en el español como L1. Biber et al., 2006, en un análisis de la frecuencia de 2.085.990 formas verbales en un corpus de español que contiene más de 21 millones de palabras de muestras de español hablado y escrito por hablantes nativos de una variada gama de registros constatan que, tanto en la lengua oral como escrita, la proporción de formas del modo subjuntivo utilizadas por los hablantes nativos es pequeña en comparación con otras conjugaciones como, por ejemplo, el pretérito indefinido y el imperfecto del modo indicativo. El análisis establece que el uso del modo subjuntivo, en los tiempos presente y pretérito, comprende sólo el 7,2\% de todas las formas verbales de la muestra. Por otra parte, Montrul (2008) demuestra que hablantes de español que adquieren de manera incompleta esta lengua (heritage speakers) son mucho más vulnerables a la atrición del modo subjuntivo que a la del indicativo en sus pretéritos indefinido e imperfecto. Si el subjuntivo es vulnerable en la competencia de este tipo de hablantes de español, no es de sorprenderse que sea particularmente difícil de adquirir por estudiantes de español como L2. Esta situación parece empeorar cuando se trata de una característica que no encuentra un correlato fácilmente transferible desde la L1 y se produce un mapeo específico entre formas morfológicas y categorías abstractas que se han constatado como de especial dificultad en la experiencia de enseñanza del ELE, y que ha convertido al modo subjuntivo en uno de los paradigmas morfológicos más distintivos, por ejemplo, para los estudiantes anglófonos de ELE.

Por otra parte, la evidencia ha demostrado que las connotaciones del subjuntivo tienen un valor comunicativo bajo (Farley 2004; VanPatten 1997), y así lo demuestran las siguientes transcripciones de conversaciones sostenidas en entrevistas informales con estudiantes extranjeros que tienen un nivel de español intermedio-avanzado (B1) y 
son potenciales candidatos a una intervención lingüística que contempla el uso de estrategias de retroalimentación correctiva en la enseñanza del presente de subjuntivo en ELE en nuestra Universidad.

\section{Trascripción:}

¿Por qué es importante para ti aprender español?

En realidad, no creo que puedo estudiar en la universidad sin que hablo bien español. Pero todavía necesito aprender mucho para que cuando yo escribo las pruebas mis profesores entienden todo que quiero decir. Además, también es bueno que aprendo bien porque cuando yo vuelvo a mi país voy a trabajar con personas latinas con problemas legales de inmigración ... así cuando ellos ven que hablo su idioma ellos tienen más confianza en mí. No quiero que piensan que sólo soy una persona para ganar plata con ellos. Es bueno que la universidad nos da clases para que aprendemos más rápido, pero en este momento todavía necesito muchos amigos chilenos que me enseñan palabras nuevas y me ayudan a practicar dichos y expresiones.

(Sujeto 9, 23 años, L1 inglés, 12 de marzo de 2011).

¿Por qué elegiste este país para venir a estudiar?

Porque me gusta mucho Chile, pero no me gusta que hay temblores ... me alegro que no ha temblado mucho en estos meses y no quiero que tiembla hasta que yo me voy ... o que mejor no vuelve a temblar nunca más aquí.... También porque la universidad es buena y hermosa, pero me da tristeza que la gente no cuida mucho la naturaleza y que tienen tantos perros solos en las calles. Espero que después que pasa algún tiempo yo me acostumbro al acento y puedo entender mejor para viajar sola y conocer más. Igual parece que no es peligroso que una mujer viaja sola en este país. Mejor si encuentro un amigo o amiga que me acompaña. Quiero conocer Torres del Paine, San Pedro de Atacama y ojalá también puedo ir a la Isla de Pascua.

(Sujeto 23, 20 años, L1 inglés, 7 de abril de 2011). 
En estas conversaciones podemos apreciar que los estudiantes se dan a entender, comunican, con relativa claridad, a pesar del escaso o nulo uso que hacen de formas de subjuntivo. Sin embargo, los propios estudiantes manifiestan que perciben el esfuerzo de sus interlocutores por entenderlos y, aunque logran desenvolverse en situaciones cotidianas, no es el nivel que ellos consideran adecuado para un buen desempeño académico.

Dada esta problemática así planteada hasta aquí en este estudio, se pretende informar desde la Lingüística Aplicada la secuencia de adquisición observada para dos estructuras del español en estudiantes extranjeros de nivel B1 en las habilidades de comprensión lectora y producción escrita. Además, se exploran algunas de las dificultades que una de estas estructuras plantea para su enseñanza y adquisición en ELE.

Sostenemos que la adquisición de los pretéritos indefinido e imperfecto del modo indicativo es claramente anterior a la adquisición del presente del subjuntivo, y afirmamos que la enseñanza del modo subjuntivo en el caso del ELE para las lenguas maternas estudiadas se ve dificultada inicialmente por la falta de notoriedad morfológica de las inflexiones que reflejan el contenido semántico-pragmático de este modo en español. En el acaso de dos de las tres lenguas estudiadas, también dificulta su enseñanza y adquisición el hecho de que en ellas la modalidad subjuntiva encuentra su expresión principalmente en mecanismos paraverbales y en contextos que no siempre coinciden con los del español. Por otra parte, según indican Isabelli y Nishida (2005), para la selección modal es necesario procesamiento sintáctico, por lo cual los estudiantes necesitan estar en un punto de su desarrollo lingüístico en que puedan confiar en su producción de cláusulas subordinadas en vez de depender exclusivamente de la coordinación $u$ otras estrategias paratácticas. A lo descrito anteriormente se suma la falta de compromiso con el valor de verdad de un enunciado en subjuntivo que aluden todos los autores al abordar el tema (Palmer 2001).

Este artículo está organizado en las siguientes secciones: en la sección 1 se presentan los descriptores teóricos del MCER que articulan algunas de las funciones de las estructuras del pretérito indefinido/imperfecto y del presente de subjuntivo españoles a nivel B1 en ELE. La sección 2 comprende el marco de referencia, en el que se reseña la enseñanza del modo subjuntivo en ELE, el orden de adquisición de una L2, la 
transferencia lingüística como estrategia de aprendizaje, de comprensión, de producción y su relación con diversos tipos de estructuras. En la sección 3 presentamos las preguntas de investigación y los objetivos que de ellas se desprenden. En la sección 4 describimos la metodología, la muestra, el instrumento y el procedimiento con que se recolectaron los datos. La sección 5 contiene los resultados y su respectivo análisis. La sección 6 presenta las conclusiones y, finalmente, en la sección 7 se incluyen las referencias bibliográficas.

\section{Marco de referencia}

La investigación en ASL ha demostrado que los estudiantes se benefician de la enseñanza que incluye formas lingüísticas propias de la etapa siguiente en su adquisición de una segunda lengua, mientras que la intervención dirigida a etapas demasiado anteriores o posteriores al nivel de desarrollo lingüístico en la segunda lengua de los estudiantes resulta confusa o irrelevante y puede llevarlos a conductas de evitación o pérdida de confianza que, a su vez, aumentan el filtro afectivo. Pienemann en su Teoría de la "Procesabilidad" explica el desarrollo de la L2 de los estudiantes en términos de la compatibilidad de la información gramatical desde niveles de constituyentes diversos en la interlengua de los estudiantes, por ejemplo, dentro de una oración, entre oraciones y entre cláusulas. En la extensión de esta teoría de la procesabilidad, Pienemann, Di Biase \& Kawaguchi y Håkansson (2005) señalan que los estudiantes hacen la elección de una estructura sintáctica en función de la madurez de su interfaz sintáctico-pragmática.

La utilidad de la instrucción formal en el modo subjuntivo se ha visto cada vez más cuestionada a medida que los investigadores han ido notando que la eficacia de esta instrucción a nivel intermedio es, en su mayor parte, débil (Collentine 2003). Collentine sostiene que para lograr objetivos de proficiencia razonables, los estudiantes necesitan numerosas oportunidades de adquirir y automatizar previamente el conocimiento necesario para procesar estructuras verbales como, por ejemplo, el pretérito indefinido y el imperfecto del modo indicativo, según lo demostrado por el estudio de Montrul (2008). 


\subsection{La enseñanza del modo subjuntivo en ELE}

La ineficacia general de la enseñanza del subjuntivo español como lengua extranjera puede resultar de la suposición de que, cuando comienza a enseñarse este modo, los estudiantes ya pueden procesar y generar una sintaxis compleja. Sin embargo, Collentine (2010) demuestra que cuando a los estudiantes se les comienza a enseñar el subjuntivo su sintaxis española es aún inmadura, lo cual genera enunciados que son característicos de lo que Givón (1976) denomina etapa pre-sintáctica del desarrollo lingüístico.

En consecuencia, cabe preguntarse si el subjuntivo español podría ser más "aprendible" si antes de intentar enseñarlo pudiéramos determinar el grado de madurez lingüística de los estudiantes dentro de una secuencia de adquisición del ELE. De verificarse una secuencia, mediante el mayor o menor dominio que los estudiantes evidencien de las estructuras en estudio, cabría también preguntarse si es posible diseñar estrategias de feedback correctivo eficaces a partir de lo observado en relación con esa secuencia y los posibles mecanismos de aprendizaje que los estudiantes utilizan para avanzar en su adquisición. En este estudio nos hacemos cargo de la determinación del grado de desarrollo lingüístico de los sujetos sobre la base del mayor dominio observado en el caso de los pretéritos del modo indicativo en relación con el presente del modo subjuntivo a partir de lo inferido de los descriptores del MCER.

\subsection{Orden de adquisición de una L2}

Los investigadores han descubierto un orden consistente en la adquisición de la lengua materna en los niños y esto ha generado gran interés en los estudiosos de la ASL.

Se han destinado grandes esfuerzos para someter a prueba la "hipótesis de la identidad", que sostiene que la adquisición de la L1 y una L2 se ciñen a los mismos patrones (Dulay y Burt 1972). Esto no ha sido confirmado, esencialmente porque las circunstancias cognitivas y afectivas de quienes aprenden una L2 son diferentes a las de quienes adquieren una L1. Los órdenes de adquisición en ASL a menudo se asemejan a aquéllos encontrados en la adquisición de la L1 y pueden tener causas neurológicas comunes; sin embargo, no existe evidencia de esto. Por el momento, no es aconsejable afirmar que el orden de adquisición de la L1 tenga alguna implicancia fácil para la ASL. 
En el campo de la adquisición de segundas lenguas se ha discutido desde distintos enfoques teóricos la posibilidad de establecer un modelo del sistema de desarrollo gramatical que siguen los estudiantes de una lengua dada, tanto en adquisición natural como en la sala de clases. En esta línea de investigación, ya en 1966 Greenberg examinó el rol de los universales tipológicos en la adquisición de una L2 y en la instrucción de la L2. Posteriormente, estudios experimentales sobre la enseñanza de diferentes tipos de oraciones de relativo (Doughty 1988, Eckman, Bell y Nelson 1988) demostraron que alumnos que recibían enseñanza en una categoría marcada de una L2 evidenciaron haber mejorado en su conocimiento no sólo de esa categoría marcada sino también en las categorías menos marcadas.

Lo que estos estudios indican, en principio, es que la exploración de una secuencia de adquisición de las lenguas puede ser de gran utilidad para esclarecer la dificultad de adquisición de ciertas estructuras en una L2. Sin embargo, estudios lingüísticos de este tipo no abundan en la adquisición del español como lengua extranjera.

El descubrimiento de que quienes aprenden una L2 pasan a través de etapas predecibles en la adquisición ha hecho que aparezcan propuestas metodológicas para la ASL diversas. Krashen (1985), por ejemplo, propone que los profesores pueden utilizar formas lingüísticas del nivel inmediatamente siguiente (denominado $i+1$ ) con cualquier input lo suficientemente rico y significativo para los alumnos. La propuesta de Krashen incluye una prohibición virtual de enfocar la atención en las formas lingüísticas y enfatiza el valor de métodos pedagógicos basados exclusivamente en un input significativo. La hipótesis de la "aprendibilidad" postulada por Pienemann (1985) difiere de lo postulado por Krashen en que considera apropiado identificar la etapa del desarrollo lingüístico en que se encuentran los alumnos y apuntar a la siguiente etapa creando o seleccionando el material instruccional apropiado. A diferencia de Krashen, Pienemann no abordó una metodología (cómo enseñar), sino que se limitó a consideraciones sobre qué enseñar y cuándo enseñarlo. Aunque las propuestas son diferentes, ambas coinciden en que los alumnos procesan y utilizan en pos de la adquisición sólo aquello que es adecuado a su etapa de desarrollo de la segunda lengua dentro de una secuencia. 
Sin embargo, aún hoy después de muchos años, las etapas de desarrollo lingüístico que se han identificado cubren una pequeñísima fracción de sólo algunas lenguas, entre las que no se cuenta el español. Todavía no estamos en condiciones de establecer un orden de adquisición que cubra adecuadamente todo lo que los estudiantes de una L2 necesitan aprender. Además, la heterogeneidad de los tipos de instrucción es una realidad que complica aun más la manera de organizar la instrucción sobre la base del desarrollo lingüístico en la adquisición de una segunda lengua por parte de los estudiantes.

La investigación en ASL hasta el momento ha logrado demostrar que los estudiantes aprenden formas lingüísticas en una secuencia con o sin una metodología, lo cual tiende a confirmar el hecho de que las secuencias de adquisición pudieran ser el resultado de mecanismos o procesos internos de los estudiantes, más que de cualquier tipo de enseñanza al que se les exponga. Como hemos visto, la hipótesis de la identidad no ha sido confirmada, el problema de la secuencia de enseñanza de las formas lingüísticas ha sido muy poco investigado y sólo ha sido descrito en términos de algunas secuencias de desarrollo para el inglés y el alemán (Pienemann, Johnston y Brindley 1988; Pienemann 2005, 2011). Desde una perspectiva de la ASL consideramos que es importante saber si la enseñanza que no coincide con la etapa de desarrollo lingüístico de los estudiantes es necesariamente ineficaz o contraproducente, como lo indican a su modo tanto Krashen como Pienemann.

\subsection{Transferencia lingüística}

La investigación en ASL consistentemente ha demostrado que el uso y la promoción de la L1 de los estudiantes en la adquisición del inglés no sólo carecen de efectos negativos para el proceso de aprendizaje, sino que ayudan efectivamente en el desarrollo de una L2, (Thomas y Collier 1997, 1999, 2002).

Desde hace ya varias décadas se ha estudiado en detalle la transferencia lingüística. En orden cronológico, existen importantes estudios a este respecto, como, por ejemplo, los de Vildomec (1963), Gass y Selinker (1983), Kellerman y Sharwood Smith (1986), Ringborn (1987), Dechert y Raupach (1989), Odlin (1989), Sjöholm (1995), Jarvis (1998), Cook (2003), Jarvis y Pavlenko (2008). 
El término "transferencia" se refiere a la incorporación y uso de características de una lengua conocida en el sistema de una lengua que se está aprendiendo (Gass 1996). Desde la década de 1980 se utiliza el término transferencia de manera intercambiable con el término "influencia interlingüística", a pesar de que algunos investigadores no consideran adecuado utilizar transferencia debido a que se asocia con la noción conductista de transferencia de habilidades. Otro término que se utiliza con frecuencia es el de "interferencia", pero éste también tiene connotaciones del conductismo y, además, plantea la desventaja de dirigir nuestra atención sólo a resultados negativos. En 1986, Kellerman y Sharwood Smith propusieron utilizar "influencia interlingüística" como hiperónimo neutro del fenómeno que cubre toda la gama de posibilidades en que el conocimiento de una lengua puede afectar el conocimiento y el uso de otra lengua en una persona.

Existen modelos y marcos teóricos diseñados específicamente para estudiar la influencia interlingüística y la interacción de las lenguas en la mente (Cook 2003, MacWhinney 2005). En esta misma línea de investigación, desde la neurofisiología se está estudiando la manera en que las lenguas se almacenan y operan en el cerebro, especialmente en el cerebro de personas que saben y utilizan más de una lengua. La investigación neurolingüística mediante el uso de tomografía de emisión de positrones (PET), la imaginología de resonancia magnética funcional (fMRI), los registros electro y magnetoencefalográficos (EEG) y los potenciales de eventos relacionados (ERP) ya han dado a conocer varios importantes descubrimientos sobre la interacción de lenguas en el cerebro (Emmorey, 2003, Fabbro 2002, Franceschini, Zappatore y Nitsch 2003, Greene 2005, Paradis 2004, Ullman 2006). Sin embargo, esta fase en el desarrollo de la investigación sobre la influencia interlingüística no estará sólidamente consolidada sino hasta dentro de varios años más, cuando las herramientas de la investigación neurolingüística permitan una descripción más precisa de la naturaleza exacta de las representaciones morfológicas, léxicas, semánticas, sintácticas, discursivas y pragmáticas en el cerebro, y podamos entender los procesos neurolingüísticos y neurofisiológicos mediante los cuales se adquieren, almacenan y recuperan estas representaciones, junto con la manera en que las representaciones de dos lenguas diferentes se pueden asociar o incluso integrar. También es posible que algunos de estos fenómenos nunca logremos explicarlos desde un nivel neurolingüístico. 
Aunque el término "influencia interlingüística" ha ganado aceptación en el ámbito de la investigación en ASL, continúan utilizándose como sinónimos los términos "transferencia" e "interferencia". En palabras de Odlin (2007): “Transferencia es la influencia que resulta de similitudes y diferencias entre la lengua meta y cualquier otra lengua que se haya aprendido con anterioridad (aunque posiblemente de manera imperfecta)". La acepción de Odlin es la que resulta más adecuada para acotar el alcance de este estudio y es la que usaremos de aquí en adelante.

La transferencia es discernible en la producción lingüística de estudiantes de una L2 (L3, L4...) en donde algunos aspectos de la estructura (superficial o profunda) de la L1 (L2, L3...) de un hablante son sustituidos de manera constante por una forma de la L2 (L3, L4 ...) y que tiene como resultado una producción no nativa.

Se puede establecer una distinción entre transferencia negativa (inhibidora o interferencia) y transferencia positiva (facilitadora). La transferencia negativa impide manejar conocimiento nuevo y se puede producir cuando el patrón transferido es distinto (aunque a veces tenga una afinidad superficial) del patrón correspondiente de la L2, con lo cual se produce la emergencia de formas erróneas. Si el estudiante encuentra que la estructura diferente es difícil, puede tender a evitarla o producir una representación minimizada de ella; de este modo, los efectos de la L1 se manifiestan no sólo en lo que el estudiante hace (es decir, en errores) sino también en lo que no hace (Seliger, 1989).

\subsection{Transferencia como estrategia de aprendizaje}

Considerando la ASL como un proceso en el que el estudiante se involucra activamente, los mentalistas enfatizaron la importancia de la capacidad mental innata del estudiante para adquirir y procesar la lengua (en lugar de factores ambientales) y comenzaron a considerar la transferencia como una estrategia de aprendizaje. El uso de la L1 para aprender una lengua se considera así como una mera manifestación de una estrategia psicológica universal (consciente o inconsciente), una estrategia que se basa en el conocimiento existente para facilitar el aprendizaje. La L1 se debe conceptualizar debidamente como un recurso junto con la interlengua- que los estudiantes aprovechan en el proceso de formación de hipótesis de la L2 (Schachter, 1992). A modo de apoyo

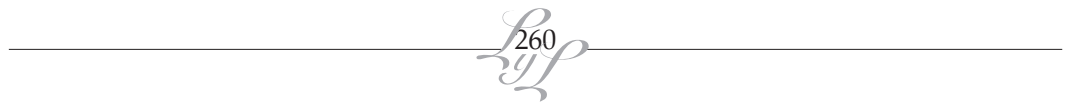


de esta afirmación está el hecho que ciertos errores de transferencia se verifican en la totalidad de poblaciones que comparten la misma L1, y también por la fosilización de ciertas estructuras influidas por la L1 en la interlengua de los estudiantes (Ellis, 1994). Con la transferencia definida como un proceso psicológico, resulta obvio que se pueden transferir sólo aquellas reglas y estructuras de las que el estudiante esté consciente (Meisel 1980). Por supuesto, esto no descarta la transferencia inconsciente de otras construcciones y principios, lo cual valida la razón de ser de la toma de conciencia selectiva y de la instrucción con la lengua como interfaz.

Sin embargo, no toda la transferencia en la comunicación necesita ser de naturaleza estratégica. Færch y Kasper (1989) distinguen tres tipos de transferencia de producción:

- Transferencia estratégica, cuando el estudiante asigna atención focal a un problema comunicativo y su solución, la cual puede involucrar el uso de la L1.

- Transferencia subsidiaria, que se produce cuando no hay conciencia focal ni del problema de producción ni de conocimiento transferido de la L1, aunque se puede desarrollar como consecuencia del monitoreo;

- Transferencia automática, que ocurre cuando el estudiante utiliza una sub-rutina altamente especializada de la L1, con la atención completamente dirigida a otros aspectos del proceso de producción.

Mientras que un estudiante más competente prefiere estrategias provenientes de la L2 (resuelve el problema usando formas de la L2) tales como la paráfrasis, un estudiante menos avanzado favorece las estrategias de reducción (abandona parte del mensaje original) o estrategias provenientes de la L1. Haastrup y Phillipson (1983) descubrieron que estas últimas son menos efectivas, puesto que consistentemente desembocan en una comprensión parcial o en un malentendido. En consecuencia, los estudiantes que usan las mejores estrategias parecen ser aquellos que cuentan con una competencia formal adecuada.

\subsection{Transferencia como producto}

Corder (1978) detestaba el concepto conductista de "interferencia", tradicionalmente percibido como característica del aprendizaje, y lo incorpora a un marco cognitivo denominándolo "intercesión", 
considerada como una estrategia de actuación activa por parte del estudiante. Los errores de "interferencia" pueden entonces no resultar de la transferencia negativa, sino de "préstamos", "el uso de un ítem de una lengua -habitualmente la materna- de naturaleza léxica o sintáctica, para subsanar las deficiencias de la interlengua". De tal forma, la transferencia no se considera una estrategia de aprendizaje, sino como una estrategia comunicativa, una técnica compensatoria sistemática para solucionar problemas de comunicación cuando no se tiene el conocimiento necesario. Mucho antes de esta conceptualización de Corder, Newmark y Reibel (1968) habían formulado la "hipótesis de la ignorancia", según la cual la interferencia de la L1 refleja ignorancia y su función principal es la de salvar brechas en la competencia del estudiante.

\subsection{Transferencia de comprensión}

La L1 del estudiante juega un rol no sólo en la producción, sino también en la comprensión de estructuras de la L2. La transferencia de comunicación involucra la comprensión y la producción, aunque esta última ha recibido mayor atención probablemente debido a que es más fácil de detectar en la actuación inicial. Como lo demuestra Ringborn (1987), la transferencia es tan importante en la comprensión como lo es en la producción, en el sentido de que ambas son inherentes a todo acto de comunicación. Sugiere que la L1 constituye "conocimiento potencial" que se puede aprovechar mejor en la decodificación (mapeo de forma a función) que en la codificación (mapeo de función a forma). Por ejemplo, la influencia de la L1 en el aprendizaje del orden sintáctico de una L2 no solo se manifiesta en la interlengua del estudiante, sino también en la recepción (interpretación) de esa L2.

Ringbom (1987) sugiere que es la transferencia de comprensión la que tiene mayor probabilidad de inducir un cambio en la interlengua del estudiante, pero la producción de la L2 también puede contribuir de manera significativa (Ellis 1994).

\section{Restricciones a la transferencia}

Se han identificado varias restricciones y factores que influyen en la transferibilidad de algunos ítems:

- El nivel lingüístico (fonología, léxico, gramática, discurso): Ellis, 1994.

- Marca: Hipótesis de Marca Diferencial de Eckman, 1984.

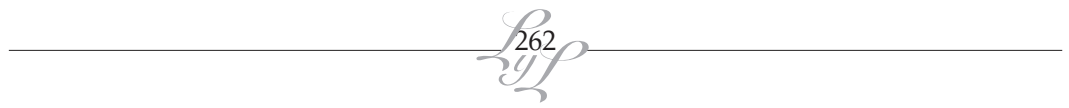


- Prototipicidad: Kellerman 1987.

- Distancia entre las lenguas y psicotipología: Swan y Smith, 2001, Callies 2006.

- Factores del desarrollo: Corder, 1978.

- Diferencias individuales: Zybert, 2007.

- Factores sociolingüísticos: Ellis, 1994.

\subsection{Transferencia y robustez de las estructuras}

La transferencia también ha sido estudiada dentro del marco Minimalista de Chomsky, 1995). Muchos enfoques generativistas actuales de la adquisición de la lengua explican por qué hay que prestar atención a la transferencia en forma selectiva: sólo afecta características de la interfaz, aquellas áreas de la gramática donde la sintaxis interactúa con la semántica o la pragmática (características interpretables) y causa opcionalidad de la L2 y atrición de la L1 en esta área (Robertson y Sorace 1999; Sorace 2000; Montrul 2004; Sánchez 2004; Tsimpli et al. 2007), en lugar de prestar atención a dominios sintácticos menores, donde sólo permanecen intactas características sintácticas (no interpretables). Esta perspectiva de los dominios vulnerables de la transferencia se ciñe a la teoría de la lingüística generativa al diferenciar las características morfosintácticas en términos de su interpretabilidad:

- Características interpretables, con representaciones semánticas, tales como tiempo y aspecto.

- Características no interpretables, puramente sistémicas, usadas para organizar la estructura y dirigir las derivaciones sintácticas, pero que carecen de contenido semántico. (Chomsky, 1995).

En la actualidad se acepta de manera general que la transferencia puede funcionar como estrategia de aprendizaje o de comunicación; sin embargo, admitimos que no es el único efecto de la influencia interlingüística en el proceso de la ASL. Resulta oportuno retomar aquí la hipótesis de la ignorancia introducida por Newmark en 1966, reformulada posteriormente por el propio Newmark y Reibel (1968) en la siguiente cita: "Para una persona que habla una L1 y está aprendiendo una L2 existen aún muchas cosas que no conoce sobre esa L2. ¿Qué puede esta persona hacer para compensar lo que no conoce, sino usar lo que sí 
conoce? A un observador que conozca la L2 le parecerá que esa persona está sustituyendo obstinadamente las reglas de la L2 por las de la L1. Sin embargo, desde el punto de vista de quien está aprendiendo, todo lo que está haciendo es desplegar su mayor esfuerzo: para salvar la brecha en su aprendizaje, recurre a lo que ya conoce."

Recientemente ha comenzado a aparecer evidencia que señala que la transferencia también se produce desde la L2 hacia la L1 (Jarvis y Pavlenko 2008). Se trata de casos en los que queda claro que ésta no es sólo un mecanismo de apoyo en la L1 cuando se carece de información sobre la L2. La situación está conformada por una compleja interacción entre la influencia interlingüística y los principios naturales de la ASL que nos obligan a replantearnos lo que sabemos sobre la transferencia desde la L1 a una L2.

Finalmente, el planteamiento de VanPatten en relación con la ASL y la L1 sostiene que cuando los estudiantes de una L2 construyen la gramática de la interlengua de esa L2 son plausibles tanto la posición que sostiene que los estudiantes recurren a la gramática universal, como la posición que sostiene que recurren a sus conocimientos de la L1. Específicamente, este estudio adhiere a la plausibilidad de que los estudiantes, al menos inicialmente, recurren a la L1 al enfrentarse a oraciones subordinadas en presente de subjuntivo en ELE.

\section{Preguntas de investigación y objetivos}

Se delimitan las siguientes dos preguntas de investigación que guían los objetivos de este estudio:

a) ¿Se observa un patrón secuencial de adquisición entre los pretéritos definido/imperfecto del modo indicativo y el presente de subjuntivo en estudiantes de ELE de nivel B1?

b) ¿Evidencian estudiantes de ELE de nivel B1 estrategias provenientes de su L1 cuando interactúan con oraciones subordinadas que en español requieren presente de subjuntivo y otro modo verbal en su L1?

En concordancia con las preguntas delimitadas para este estudio, nos planteamos los siguientes dos objetivos:

a) Revisar un segmento de la secuencia de adquisición del español como lengua extranjera en un grupo de hablantes nativos de alemán, francés

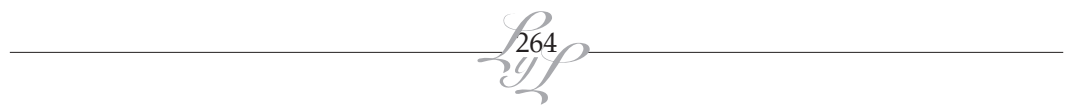


e inglés. Este segmento incluye los pretéritos indefinido e imperfecto del modo indicativo y el presente del modo subjuntivo en oraciones subordinadas. El segmento se revisará para verificar si, conforme a lo indicado por la literatura (MCER, estudios de Collentine, 2010), se evidencia un mayor grado de dominio de las primeras estructuras (pretéritos del modo indicativo) en relación con la segunda estructura (presente de subjuntivo).

b) Observar tendencias atribuibles a transferencia desde la L1 en la adquisición del subjuntivo en ELE, como respuesta tentativa a la situación planteada por VanPatten, 2007.

\section{Metodología}

Se realizó un estudio transversal exploratorio del grado de dominio de conocimiento declarativo en habilidad lectora y producción escrita del pretérito imperfecto/indefinido del modo indicativo y del presente de subjuntivo español.

\subsection{Muestra}

25 estudiantes universitarios de pre y postgrado, cuyas lenguas maternas son el alemán ( 8 sujetos), el francés ( 8 sujetos), y el inglés ( 9 sujetos). El rango etario va de los 20 a los 35 años. Cinco sujetos de sexo masculino y 20 de sexo femenino. Todos los sujetos manifestaron haber comenzado a estudiar español después de los 15 años de edad.

\subsection{Instrumento}

En concordancia con el primer objetivo de este estudio exploratorio, preparamos un instrumento que consta de cuatro secciones para la recolección de los datos. La primera sección del instrumento consiste en una narración que incluye 60 alternativas múltiples de las que los sujetos deben decidir entre el uso de pretérito indefinido o imperfecto del modo indicativo en 30 enunciados que pretenden medir el dominio de esta estructura a nivel de la habilidad de comprensión lectora.

Ejemplo (Extracto):

Instrucciones: encierra en un círculo la forma verbal que consideres más apropiada. 


\begin{tabular}{|l|l|}
\hline 5. Como ya___ mediodía, tenían & 6. Los niños no pudieron esperar \\
hambre... & más y se___ a comer ... \\
A. fue & A. se sentaron \\
B. era & B. se sentaban \\
\hline
\end{tabular}

La segunda sección del instrumento consiste en dos narraciones que incluyen 66 infinitivos verbales que los sujetos deben conjugar en pretérito indefinido o imperfecto del modo indicativo con la finalidad de medir el dominio de esta estructura a nivel de la habilidad de producción escrita. Cinco profesores universitarios hablantes nativos de español realizaron la validación del instrumento.

Ejemplo (Extracto):

Instrucciones: escribe en pasado la forma verbal dada entre paréntesis.

El conductor (sonreírse) pero no me (decir) nada. Mientras tanto, mi amigo (ir)

a buscar un taxi. El taxista (llegar) (preguntar) en seguida y nos el nombre de un hotel recomendado por una compañera de clase, pero el taxista no (parecer) entendernos. Mi amigo (repetir) el nombre del hotel y el taxista (indicar)

que ahora sí (comprender)

En los todos los casos se obtuvo un 100\% de coincidencia en las respuestas dadas por los profesores que validaron el instrumento.

La tercera sección del instrumento está diseñada para medir el dominio del presente de subjuntivo a nivel de la habilidad de comprensión lectora; consta de 3 textos que contienen tres situaciones contextualizadas. En 68 enunciados, el $52 \%$ de las formas correctas corresponden a presente de subjuntivo y el $48 \%$ corresponde a presente de indicativo. Las 272 formas verbales de las alternativas múltiples incluyen presente de indicativo, presente de subjuntivo, futuro simple de indicativo, condicional simple e infinitivo. Los verbos que requieren subjuntivo se encuentran en 
contextos de oraciones subordinadas sustantivas en función de objeto, adverbiales de tiempo introducidas por "cuando", y en el contexto de la fórmula Ser+adjetivo(masculino)+que.

Ejemplo (Extracto):

Instrucciones: Subraya la forma verbal que consideres más adecuada al contexto.

\begin{tabular}{|c|c|c|c|}
\hline \multicolumn{4}{|l|}{ ¡Toc, toc! } \\
\hline \multicolumn{4}{|c|}{ PROFESOR: ¡Adelante! } \\
\hline \multicolumn{4}{|c|}{ DELEGADA: ¡Hola! ¿ (1. Poder, yo) entrar? } \\
\hline \multicolumn{4}{|c|}{ PROFESOR: Sí, sí, Laura, adelante. } \\
\hline \multicolumn{4}{|c|}{$\begin{array}{l}\text { DELEGADA: Mira, (2. querer, yo) hablar contigo porque ( } 3 \text {. tener, } \\
\text { nosotros) un problema. }\end{array}$} \\
\hline \multicolumn{4}{|c|}{$\begin{array}{l}\text { PROFESOR: ¡Vaya por Dios! ¡Qué raro! Es extraño que (4.tener, ustedes) } \\
\text { problemas. ¿Qué pasa ahora? }\end{array}$} \\
\hline \multirow{4}{*}{\multicolumn{4}{|c|}{$\begin{array}{l}\text { DELEGADA: Es que (5. tener, nosotros) dos exámenes el mismo día y } \\
\text { (6. necesitar, nosotros) que ( } 7 \text {. cambiar, tú) la fecha del tuyo, porque el } \\
\text { otro profesor no (8. querer) oír hablar del tema. } \\
\text { PROFESOR: ¡Siempre igual! } \\
\text { DELEGADA: Sí, ya lo sé. Es lamentable que siempre (9. estar, nosotros) } \\
\text { pidiéndote favores, pero el otro profesor... (Continúa) }\end{array}$}} \\
\hline & & & \\
\hline & & & \\
\hline & & & \\
\hline \multicolumn{4}{|l|}{ Alternativas: } \\
\hline 1. Pueda & Puedo & Poder & Podré \\
\hline 2. quiero & querré & querer & quiera \\
\hline 3. tendremos & tengamos & tener & tenemos \\
\hline 4. tienen & tengan & tener & tendrán \\
\hline 5. tengamos & tendremos & tener & tenemos \\
\hline 6. necesitaremos & necesitemos & necesitar & necesitamos \\
\hline 7. cambies & cambias & cambiar & cambiarás \\
\hline 8. quiera & quiere & querer & querrá \\
\hline 9. estamos & estemos & estar & estaremos \\
\hline
\end{tabular}

La cuarta sección del instrumento consiste en 75 oraciones (25 en cada lengua extranjera) que los sujetos deben traducir al español con la finalidad de medir el dominio del subjuntivo a nivel de la habilidad 
de producción escrita. En estas 75 oraciones, el 12\% de los verbos corresponde a presente de indicativo y el $88 \%$, a presente de subjuntivo en español. Los verbos que requieren subjuntivo en español se encuentran en contextos de oraciones subordinadas sustantivas en función de objeto, adverbiales de tiempo introducidas por "cuando", y en el contexto de la fórmula Ser+adjetivo(masculino)+que. Cuatro profesores de idiomas extranjeros, hablantes nativos de las lenguas maternas en estudio y competentes en español, validaron el instrumento. Entre los cuatro profesores se obtuvo un $96 \%$ de coincidencia en las respuestas. Se produjo discusión en algunos contextos (4 casos) que podrían interpretarse como de alternancia indicativo-subjuntivo y que claramente apuntan a factores pragmáticos y contextuales presentes en la elección de la modalidad en el momento de la comunicación. Estos contextos fueron eliminados.

Ejemplo (Extracto):

Instrucciones: traduce al español las siguientes oraciones.

3. Il s'occupera du reste quand il sera dans le train.

9. I suggest that you go immediately.

15. Ich bestehe darauf, dass er kommt.

\subsection{Procedimiento}

Se aplicó el instrumento en tres sesiones diferidas de 60 minutos de duración cada una. La primera sesión consistió en la aplicación de las narraciones para el caso de los pretéritos indefinido e imperfecto del modo indicativo, en sus modalidades de selección múltiple y conjugación verbal. La segunda sesión consistió en la aplicación de las situaciones para el caso del presente de subjuntivo en la modalidad de selección múltiple. La tercera sesión consistió en la aplicación del instrumento

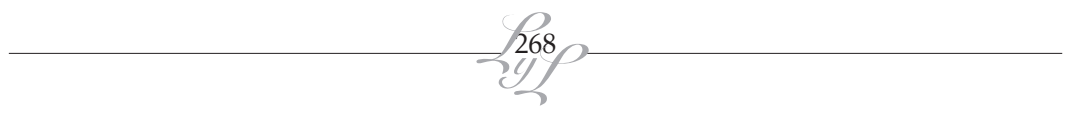


que contempla la traducción de 25 oraciones que contienen verbos en presente de indicativo y de subjuntivo desde las lenguas maternas de los sujetos al español. Se permitió el uso de diccionarios para búsqueda de palabras que no incluyera la conjugación verbal. Cada sección contiene instrucciones detalladas por escrito de lo que los sujetos deben hacer. En las tres sesiones todos los sujetos entregaron el instrumento contestado completamente dentro del tiempo proporcionado. El criterio de corrección considera como error aquellos casos en que los sujetos seleccionan o conjugan formas del modo indicativo $\mathrm{u}$ otra forma verbal en contextos que requieren el presente de subjuntivo, y aquellos casos en que los sujetos seleccionan o conjugan alguna forma verbal de subjuntivo en contextos que no la requieren.

\section{Análisis de los resultados}

En el análisis de los resultados obtenidos de los sujetos hablantes de las tres lenguas maternas de la muestra se pueden apreciar algunas tendencias. Una de estas tendencias observadas contiene la respuesta a nuestra primera pregunta de investigación. Sólo tres sujetos (alemán, sujetos 3 y 8 ; inglés, sujeto 9), evidencian porcentajes de logro superior en el presente de subjuntivo, mientras que 22 sujetos evidencian un porcentaje de logro mayor en los pretéritos de indicativo. Se observa mayor conocimiento declarativo por escrito del pretérito indefinido/ imperfecto del modo indicativo que del presente del modo subjuntivo. Este resultado es coherente con lo indicado por Collentine (2010) y Montrul (2008), y con el patrón de adquisición que se infiere a partir de los descriptores del MCER. 
Tabla 1: Porcentajes de logro indicativo/subjuntivo:

\begin{tabular}{|c|c|c|c|}
\hline Sujetos & Edad & $\begin{array}{c}\% \text { logro Indef/ } \\
\text { Imperf. }\end{array}$ & $\%$ logro Subj. \\
\hline \multicolumn{4}{|c|}{ Alemán } \\
\hline Sujeto 1 & 30 & $99 \%$ & $95 \%$ \\
\hline Sujeto 2 & 23 & $86 \%$ & $53 \%$ \\
\hline Sujeto 3 & 27 & $76 \%$ & $80 \%$ \\
\hline Sujeto 4 & 24 & $93 \%$ & $80 \%$ \\
\hline Sujeto 5 & 23 & $80 \%$ & $54 \%$ \\
\hline Sujeto 6 & 30 & $100 \%$ & $100 \%$ \\
\hline Sujeto 7 & 24 & $88 \%$ & $65 \%$ \\
\hline Sujeto 8 & 24 & $94 \%$ & $97 \%$ \\
\hline \multicolumn{4}{|c|}{ Francés } \\
\hline Sujeto 1 & 27 & $73 \%$ & $43 \%$ \\
\hline Sujeto2 & 23 & $75 \%$ & $40 \%$ \\
\hline Sujeto 3 & 22 & $64 \%$ & $39 \%$ \\
\hline Sujeto 4 & 22 & $91 \%$ & $42 \%$ \\
\hline Sujeto 5 & 34 & $89 \%$ & $67 \%$ \\
\hline Sujeto 6 & 28 & $73 \%$ & $53 \%$ \\
\hline Sujeto 7 & 29 & $96 \%$ & $60 \%$ \\
\hline Sujeto 8 & 30 & $93 \%$ & $32 \%$ \\
\hline \multicolumn{4}{|c|}{ Inglés } \\
\hline Sujeto 1 & 25 & $92 \%$ & $53 \%$ \\
\hline Sujeto 2 & 20 & $65 \%$ & $42 \%$ \\
\hline Sujeto 3 & 20 & $43 \%$ & $25 \%$ \\
\hline Sujeto 4 & 33 & $100 \%$ & $89 \%$ \\
\hline Sujeto 5 & 30 & $100 \%$ & $100 \%$ \\
\hline Sujeto 6 & 23 & $92 \%$ & $30 \%$ \\
\hline Sujeto 7 & 22 & $72 \%$ & $30 \%$ \\
\hline Sujeto 8 & 30 & $78 \%$ & $57 \%$ \\
\hline Sujeto 9 & 28 & $65 \%$ & $71 \%$ \\
\hline \multicolumn{2}{|c|}{ Promedio General } & $83,08 \%$ & $59,88 \%$ \\
\hline
\end{tabular}


La comprobación de que el pretérito indefinido/imperfecto del modo indicativo se adquiere antes que el presente de subjuntivo, según lo demuestran los resultados de este estudio, nos permite disponer de un punto de referencia documentado desde el cual determinar el grado de desarrollo lingüístico de los estudiantes antes de proceder a aplicar la parte del instrumento que mide el conocimiento declarativo en las habilidades de comprensión lectora y producción escrita del presente del modo subjuntivo con ciertos tipos de construcciones.

Para garantizar una relativa uniformidad en el grado de desarrollo lingüístico, no se consideraron los sujetos que obtuvieron menos de 50\% de logro en la prueba de la habilidad lectora del pretérito indefinido/ imperfecto del modo indicativo. Este criterio de selección redujo la muestra de 42 a 25 sujetos.

En concordancia con el segundo objetivo, se presentan a continuación los errores evidenciados para el caso del presente de subjuntivo, y de la distribución general de ellos se observa una tendencia: la de su concentración en un tipo determinado de oraciones en las tres lenguas en cuestión. Como se muestra en la tabla 2, el porcentaje de concentración de errores en un tipo de oraciones corresponde al doble del porcentaje del promedio de concentración de errores en los otros tipos de estructuras.

Tabla 2: Porcentajes de la distribución general de errores por estructura en las tres lenguas: 25 sujetos

\begin{tabular}{|l|l|}
\hline 1. Subordinadas sustantivas & $26 \%$ \\
\hline 2. Subordinadas adverbiales temporales & $52 \%$ \\
\hline 3. Expresiones con ser+adjetivo+que & $22 \%$ \\
\hline
\end{tabular}

\subsection{Distribución de errores según la L1}

Una segunda tendencia se observa en la concentración de errores dependiente de la lengua materna de los sujetos. Los errores en las tres estructuras se distribuyen de manera uniforme en un promedio de alrededor del 33\% en inglés y alemán. Sin embargo, en el caso del francés los errores en oraciones subordinadas sustantivas y en la expresión 
formulaica ser+adjetivo sólo promedian alrededor de la sexta parte del porcentaje total de errores. Por el contrario, los errores en las oraciones subordinadas adverbiales de tiempo se quintuplican en relación con las otras categorías, como se observa en la tabla 3. Estas diferencias entre las tres lenguas obedecerían al hecho de que el francés, al igual que el español y a diferencia del inglés y el alemán, cuenta con una morfología del modo subjuntivo que difiere de las marcas morfológicas del modo indicativo. Vale decir, los estudiantes francófonos ya han notado en su propia lengua el componente morfológico que conlleva el cambio modal. Los casos que registran errores en francés dicen relación principalmente con oraciones subordinadas sustantivas afirmativas con el verbo esperar, que en español utiliza subjuntivo y en francés, indicativo. En todos los errores detectados en los estudiantes francófonos en este aspecto se observa el uso de indicativo, ya sea en presente o futuro simple, después del verbo esperar en este tipo de oraciones, pero no en oraciones negativas ni interrogativas, en las que el francés suele coincidir con el español en el uso de subjuntivo.

Tabla 3: Distribución de errores según la L1:

\begin{tabular}{|l|l|}
\hline \multicolumn{2}{|c|}{ Alemán (8 sujetos) } \\
\hline 1. Subordinadas sustantivas & $34 \%$ \\
\hline 2. Subordinadas adverbiales, temporales & $35 \%$ \\
\hline 3. Expresiones con ser+adjetivo+que & $31 \%$ \\
\hline \multicolumn{2}{|c|}{ Francés(8 sujetos) } \\
\hline 1. Subordinadas sustantivas & $10 \%$ \\
\hline 2. Subordinadas adverbiales, temporales & $85 \%$ \\
\hline 3. Expresiones con ser+adjetivo+que & $5 \%$ \\
\hline \multicolumn{2}{|c|}{ Ingetos) } \\
\hline 1. Subordinadas sustantivas & $35 \%$ \\
\hline 2. Subordinadas adverbiales, temporales & $35 \%$ \\
\hline 3. Expresiones con ser+adjetivo+que & $30 \%$ \\
\hline
\end{tabular}

La alta concentración de errores en las subordinadas adverbiales temporales en el caso de los sujetos francófonos es consistente con el uso del futuro simple de indicativo en esta lengua en contextos en que el español recurre a presente de subjuntivo para este tipo de oraciones.

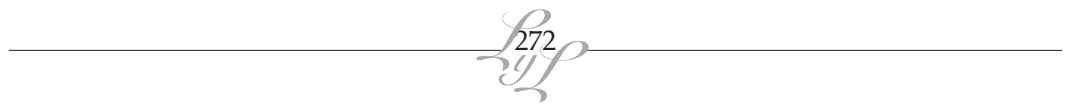


El hecho de que en los sujetos cuyas lenguas maternas son el alemán y el inglés los promedios de error en las 3 categorías se distribuyan de manera más uniforme que en francés indica una dificultad generalizada de estos sujetos en relación con el presente de subjuntivo español. En estas dos lenguas, la modalidad no encuentra su expresión precisamente en la morfología verbal. Existen en inglés y alemán construcciones formulaicas y mecanismos paraverbales que expresan la modalidad subjuntiva, además de indicios de marca morfológica que no necesariamente coinciden con los contextos de uso del subjuntivo español. De acuerdo con la Hipótesis de Marca Diferencial postulada por Eckman (1984), las reglas esenciales de la L2 (versus las marcas periféricas, reglas idiosincrásicas), las formas y los universales menos marcados que las características equivalentes en la L1 se pueden considerar más transferibles. Según Eckman, las áreas de dificultad que un estudiante de una L2 tendrá pueden predecirse mediante la comparación sistemática de la L1, la L2 y las relaciones de marca establecidas en la gramática universal.

La idea detrás del concepto de marca lingüística indica que las oposiciones entre ciertas representaciones lingüísticas no son simplemente opuestos polares, sino, más bien, se considera que un miembro de una oposición es privilegiado, en el sentido de que tiene una distribución más amplia, tanto dentro de una lengua y también entre varias lenguas. Conceder un valor de marca a esta oposición es la forma de caracterizar esta condición: el miembro con mayor distribución se designa como no marcado, con lo cual se indica que es más básico y más natural que el otro miembro, definido como "marcado". La distribución en la que existe una relación de implicación entre la ocurrencia de los miembros de una oposición se conoce como "marca tipológica", concepto desarrollado por Greenberg (1966), que define como marcada tipológicamente una estructura $X$ en relación a otra, $Y$, (e $Y$ no tiene marca tipológica en relación a $X)$, si una lengua que tiene $X$ también tiene $Y$, pero la lengua que tiene $Y$ no necesariamente tiene $X$.

Desde esta perspectiva, la marca tipológica es una relación asimétrica, irreflexiva y transitiva entre las representaciones lingüísticas de las lenguas del mundo, de manera tal que la presencia de una estructura en una lengua implica la presencia de otra estructura, pero no viceversa.

El subjuntivo francés resulta ser notoriamente menos marcado (más natural y co-ocurrente) que el del inglés y el alemán en relación con el 
subjuntivo español. Particularmente en el presente del tipo de oraciones estudiadas, en francés en las oraciones subordinadas sustantivas sólo difiere del español el uso de subjuntivo con el verbo esperar, que en francés utiliza indicativo (presente o futuro). En inglés y alemán para este tipo de oraciones existe también la posibilidad de marca morfológica que, sin embargo y debido a su baja frecuencia en el caso del alemán y la casi imperceptible falta de la inflexión -s en el caso de la tercera persona en inglés, esta marca parece no ser suficiente para que los estudiantes establezcan un punto de partida al abordar el aprendizaje de este modo español. Por ejemplo, aunque en un contexto de uso que no coincide con el del español, en el discurso indirecto alemán existe la posibilidad de usar un subjuntivo con una marca morfológica que difiere claramente de la de indicativo. Así, frente a una oración como "Él afirma que está enfermo", en alemán tenemos dos posibilidades: "Er behauptet, dass er krank sei/ist". Formas como éstas subsisten aún en la lengua alemana (bekäme, fände, gäbe, ginge, nähme, etc.) y a menudo involucran el valor de verdad concedido por el hablante respecto de lo dicho por un tercero. En nuestro estudio los sujetos invariablemente tienden a sustituirlas con la perífrasis würden + infinitivo, situación que los lleva a confundir el subjuntivo con el condicional español, con la consecuente dificultad que de ello surge para la enseñanza del subjuntivo español. El punto que tratamos de exponer es que, aunque en un contexto de uso distinto al del español, la marca morfológica de subjuntivo existe en alemán. Si bien es cierta esta delimitación entre ambas formas (Konjunktiv I/ Konjunktiv II), se observa cada vez más en alemán una tendencia a la desaparición del KI (el cual contiene marcas morfológicas que difieren de las formas de indicativo), que va quedando relegado casi exclusivamente al plano escrito de la lengua, en tanto que en el hablado se usa cada vez más la forma analítica würden + infinitivo. La totalidad de los sujetos enfrentados a la traducción de este tipo de oraciones del español al alemán optó por la perífrasis würden + infinitivo. Al consultarles sobre la otra posibilidad, la mayoría admitió conocerla, pero la consideraban "anticuada".

En inglés, la morfología del presente del modo subjuntivo coincide con la del indicativo, excepto en la tercera persona de singular. Tenemos así, por ejemplo: Ellos vienen $=$ They come; Él viene $=$ He comes. Exigimos que ellos vengan $=$ We demand that they come; Exigimos que él venga $=$ We demand that he come. Existen también algunas expresiones formulaicas que en inglés usan subjuntivo: God save the Queen, Come

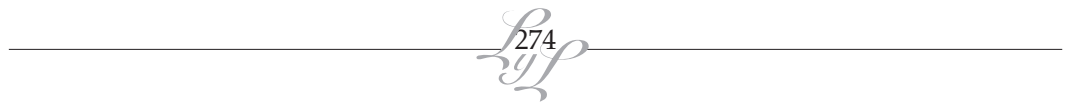


what may, etc. Con todo, tanto para el inglés como para el alemán, estas instancias no parecen servir de guía a los estudiantes al momento de abordar el subjuntivo español, que en relación con estas dos lenguas resulta más "marcado".

La construcción formulaica ser+adjetivo+que registra la tendencia más baja a errores en las tres lenguas, con un promedio de $25 \%$ en inglés y alemán. Los errores en esta misma construcción registran sólo una quinta parte de ese promedio en el caso de los sujetos cuya lengua materna es el francés, probablemente debido a las mismas razones expuestas para el caso de las oraciones subordinadas sustantivas: distancia entre las lenguas (Swan y Smith, 2001); en francés se usa subjuntivo en este tipo de oraciones. Sin embargo, la uniformidad de los porcentajes de error del patrón formulaico ser+adjetivo+que con los porcentajes de error de los otros dos tipos de oraciones en dos de las tres lenguas no es consistente, en principio, con la teoría que sugiere que la vulnerabilidad de la interfaz (sintáctico-pragmática, en la teoría modular) se puede mejorar con instrucción explícita de la pragmática del subjuntivo, en lugar de hacer que los estudiantes establezcan asociaciones léxicas o de patrones como, por ejemplo, "ser + adjetivo (masculino) + que" + subjuntivo. A pesar de que no podemos dar cuenta de la manera en que los sujetos del estudio obtuvieron el conocimiento que demuestran tener sobre el presente de subjuntivo español, es lógico suponer que, hayan o no recibido instrucción formal previa en ELE, el conocimiento que demuestran de este tipo de patrón procede de una instancia de aprendizaje similar o idéntica (implícita o explícita) a aquella mediante la cual adquirieron conocimiento sobre los otros dos tipos de estructuras. Si los errores se distribuyen en tipos de oraciones distintos que dependen de la L1, entonces resulta evidente que para enseñar el presente de subjuntivo en ELE se necesitan estrategias de enseñanza y retroalimentación correctiva diferenciadas.

\subsection{Logro en actuación en comprensión lectora versus producción escrita}

Una cuarta tendencia observable nos indica que en ambas estructuras el logro es mayor en las respuestas de selección múltiple (comprensión lectora) en comparación con las respuestas en que los sujetos debieron elaborar mediante conjugación la forma verbal, los pretéritos del modo indicativo y traducción de las formas del presente de subjuntivo 
(producción escrita), respectivamente. Esta diferencia dependiente del tipo de modalidad de respuesta la retomaremos en objetivos ulteriores específicos de nuestro estudio de la secuencia de adquisición del ELE y la sincronía con la etapa de desarrollo lingüístico de los estudiantes, ya que involucra distinciones entre competencia y actuación y es de importancia para el tipo de retroalimentación correctiva recomendable para cada caso.

Tabla 4: Porcentajes de logro en actuación guiada versus actuación libre.

\begin{tabular}{|c|c|}
\hline \multicolumn{2}{|c|}{$\%$ Logro Promedio Indefinido/Imperfecto 25 sujetos } \\
\hline Comprensión lectora & Producción escrita \\
\hline $\mathbf{8 3 , 0 8} \%$ & $76,0 \%$ \\
\hline \% Logro Promedio Presente Subjuntivo 25 sujetos \\
\hline Comprensión lectora & Producción escrita \\
\hline $64 \%$ & $53,72 \%$ \\
\hline
\end{tabular}

Como respuesta a la plausibilidad de las dos posiciones planteadas por VanPatten (2007) y a nuestra segunda pregunta de investigación, un análisis contrastivo entre el español y cada una de las lenguas de las que provienen los errores nos permite observar interferencia, transferencia negativa, desde la lengua materna.

Del análisis del tipo de errores evidenciados en la interlengua de los 25 sujetos hablantes nativos de las tres lenguas de este estudio, junto con el análisis de las 135 oraciones traducidas al español por ellos, se observa que, cuando cometen errores en contextos de presente de subjuntivo español, el 100\% de esos errores son atribuibles a estructuras provenientes de sus lenguas maternas: observamos transferencia negativa o interferencia.

Los tipos de errores detectados, en general, son comunes a las tres lenguas, aunque tienden a distribuirse de manera diferente, dependiendo, como ya hemos visto, de la clase de oraciones en que aparecen.

\subsection{Ejemplos de los tipos de errores de transferencia provenientes de las tres lenguas del estudio}

Los errores muestran oraciones en que se usa indicativo en casos en que en español se requiere subjuntivo y también, inversamente, oraciones en que se usa subjuntivo en casos en que el español requiere indicativo,

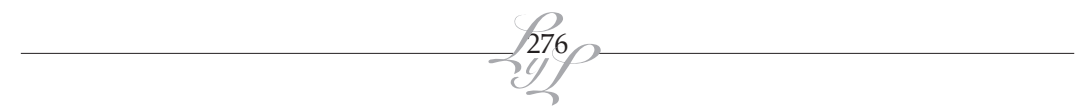


según el uso correcto que se hace de estos dos modos en las respectivas lenguas maternas.

\begin{tabular}{|l|l|l|}
\hline Te quiero & para venir & a mi fiesta*. \\
\hline I want you & to come & to my party. \\
\hline
\end{tabular}

\begin{tabular}{|l|l|l|}
\hline Él me exige & de ir & al cine* \\
\hline Il m'exige & d'aller & au cinéma. \\
\hline
\end{tabular}

\begin{tabular}{|l|l|l|}
\hline Ella & exige & que él viene. $^{*}$ \\
\hline Sie & verlangt, & dass er kommt. \\
\hline
\end{tabular}

\begin{tabular}{|l|l|l|}
\hline Necesito & que él va & a hablar con ellos. \\
\hline I need & that he goes & to talk to them. \\
\hline
\end{tabular}

\begin{tabular}{|l|l|l|}
\hline Ella dice & que tenga & sólo 85 años. $^{*}$ \\
\hline Sie sagt, & sie sei & nur 85 Jahre alt. \\
\hline
\end{tabular}

\begin{tabular}{|l|l|l|}
\hline Lo hará & cuando está/estará & en el tren. ${ }^{*}$ \\
\hline $\begin{array}{l}\text { Er wird es machen/ } \\
\text { macht es }\end{array}$ & wenn er auf dem Zug & ist. \\
\hline Il le fera & quand il sera & dans le train. \\
\hline He'll do it & when he is & on the train. \\
\hline
\end{tabular}

\begin{tabular}{|l|l|l|}
\hline Esperamos & que viene/vendrá & mañana.* \\
\hline We hope & that he'll come/comes & tomorrow. \\
\hline Wir hoffen, & dass er morgen & kommt. \\
\hline Nous espérons & qu'il viendra/vient & demain. \\
\hline
\end{tabular}

Estos resultados responden nuestra segunda pregunta de investigación, y reafirman la problemática planteada por Van Patten (2007). Sin embargo, los resultados generales de nuestro estudio, junto con evidenciar tendencias a cometer errores en principio atribuibles a la lengua materna de los sujetos, también muestran variabilidad en los 
casos en que los sujetos de las tres lenguas seleccionan y utilizan formas correctas del subjuntivo español en contextos que no coinciden con el patrón de sus lenguas maternas. En la mayoría de los casos las formas utilizadas correctamente corresponden a formas en que la morfología difiere marcadamente de la de las formas de indicativo, como por ejemplo sepas, vengas, seas, digas, etc. Esta variabilidad nos obliga a reconsiderar las posibilidades de la transferencia y de otras eventuales estrategias de aprendizaje en la adquisición tardía de esta característica del ELE.

\section{Conclusiones}

Para resumir lo que hemos descubierto en este estudio exploratorio, reiteramos algunas de las observaciones realizadas a los datos y replanteamos brevemente algunas de las nociones expuestas en el marco de referencia.

El descubrimiento de que quienes aprenden una L2 pasan a través de etapas en la adquisición ha hecho que aparezcan diversas propuestas para la ASL que indican que la metodología de enseñanza dirigida a etapas demasiado anteriores o posteriores al nivel de desarrollo lingüístico en la segunda lengua de los estudiantes resulta confusa o irrelevante. Sin embargo, aún hoy después de muchos años de estudios (Krashen 1985, Pienemann, Johnston y Brindley 1988, Pienemann, Di Biase y Kawaguchi, 2009, Pienemann 2005, 2011), las etapas que se han identificado sólo cubren una pequeñísima fracción del inglés y el alemán y aun mucho menos de otros idiomas. Todavía no estamos en condiciones de establecer un orden de adquisición que cubra adecuadamente todo lo que los estudiantes de una L2 necesitan aprender, particularmente en el caso del ELE. En nuestro estudio hemos verificado que el patrón de adquisición de las formas lingüísticas revisadas es consistente con las recomendaciones del MCER para el ELE en la secuenciación de estas estructuras. La corroboración de que el pretérito indefinido/imperfecto del modo indicativo se adquiere antes que el presente de subjuntivo, según lo demuestran los resultados de este estudio, nos permite disponer de un punto de referencia documentado desde el cual determinar el grado de desarrollo lingüístico de los estudiantes antes de proceder a enseñarles el presente del modo subjuntivo con determinados tipos de construcciones. Esto hace posible abordar el problema advertido por Collentine (2010) que señala que cuando a los estudiantes se les comienza a enseñar el subjuntivo en ELE su sintaxis española es aún inmadura, lo 
cual da como resultado una enseñanza ineficaz, pues se parte desde la falsa suposición de que los estudiantes ya pueden procesar y generar una sintaxis compleja.

Luego de haber verificado ese patrón de adquisición, mediante el mayor o menor dominio que los estudiantes demostraron de las estructuras estudiadas, y de esa forma haber dado respuesta a nuestra primera pregunta de investigación, estamos en mejores condiciones para abordar la enseñanza del presente de subjuntivo desde una medición confiable, avalada por una base empírica que nos permite proceder en sincronía con el grado de desarrollo lingüístico evidenciado por los estudiantes y de apoyar mediante retroalimentación correctiva pertinente los mecanismos de aprendizaje que ellos utilizan para avanzar en su adquisición.

En relación con los mecanismos de aprendizaje y adquisición del subjuntivo en ELE, recogimos la propuesta de VanPatten (2007) que sostiene que cuando los estudiantes de una L2 construyen la gramática de la interlengua de esa L2 es plausible que recurran a la gramática universal o a sus conocimientos de la L1 y adherimos a la plausibilidad de que los estudiantes, al menos inicialmente, recurren a la L1 al enfrentarse a oraciones subordinadas en presente de subjuntivo en ELE. Para ello, planteamos la pregunta:

¿Evidencian estudiantes de ELE de nivel B1 estrategias provenientes de su L1 cuando interactúan con oraciones subordinadas que en español requieren presente de subjuntivo y otro modo verbal en su L1?

Respondimos esta pregunta mediante un análisis contrastivo entre el español y las lenguas involucradas con el que pudimos detectar estrategias provenientes de la L1 en la producción de los estudiantes. Al comparar las oraciones erróneas en español con sus respectivas versiones (correctas) en las lenguas maternas de los estudiantes, quedan expuestos al trasluz textos escritos en dos lenguas diferentes en los que se constatan calcos idénticos de las estructuras de la L1 aplicados a la producción en la L2, como se ejemplifica en la sección de análisis de los resultados. Vale decir, los alumnos transfieren sobre la base de lo que conocen.

Expusimos que la investigación sobre ASL ha demostrado que el uso de la L1 de los estudiantes en la adquisición de una L2 no sólo carece de efectos negativos para el proceso de aprendizaje, sino que ayuda 
efectivamente en el desarrollo de una L2 (Thomas y Collier 1997, 1999, 2002); definimos el concepto de transferencia desde un marco teórico determinado y revisamos a través de un recorrido histórico la situación conceptual y teórica de la transferencia en la ASL como estrategia de aprendizaje desde el punto de vista de la comprensión lectora y la producción escrita de una L2.

Gran parte de lo observado en relación con la segunda pregunta que guía nuestro estudio se puede explicar mediante contribuciones de la investigación en ASL. Así por ejemplo, la concepción de la interlengua sugiere que la transferencia es más evidente en estados incipientes del desarrollo lingüístico y que algunos errores disminuyen a medida que aumenta el conocimiento de la L2. Sin embargo, en estos sujetos, de nivel B1, podemos apreciar transferencia negativa (interferencia) en una etapa posterior, no incipiente de su desarrollo lingüístico. De esta situación rinde debida cuenta la noción de factores del desarrollo dada por Corder (1978). Ellis (1994) plantea que la influencia de la L1 es menos prevalente en el nivel lingüístico de la sintaxis debido al mayor nivel de conciencia metalingüística de las propiedades gramaticales en la mayoría de los estudiantes. En los sujetos de este estudio, sin embargo, no observamos ese nivel de conciencia metalingüística que podría permitirles ejercer mayor control sobre la forma lingüística en cuestión, con lo cual debiera disminuir la transferencia, si nos atenemos a lo indicado por Ellis. De la noción de Prototicipidad de Kellerman (1987), resultaría interesante estudiar las percepciones que tienen los estudiantes sobre las estructuras de su propia lengua que influyen en lo que ellos transfieren y que reflejarían lo que consideran prototípico y semánticamente transparente, transferible, en su L1. A pesar de que nuestro estudio versa sobre lenguas indoeuropeas en las que la distancia entre ellas y su tipología no difiere significativamente, como sería el caso entre lenguas más distantes del español, la distancia real entre las lenguas es un factor en la transferencia positiva y negativa. Por ejemplo, finlandeses que hablan sueco y aprenden inglés cuentan con ventajas en comparación con finlandeses que hablan finlandés, aunque los últimos manifiestan menos transferencia negativa. Así, donde la L1 del estudiante tiene una característica equivalente a la de la L2, el aprendizaje por lo general se facilita. Sin embargo, la distancia percibida entre las lenguas puede ser de mayor importancia. El concepto de psicotipología como factor no estructural que juega un rol en la transferencia denota 
la noción que percibe el estudiante de la cercanía entre la L1 y la L2. De tal manera, los hablantes de lenguas que no se relacionan entre ellas se enfrentarán a problemas que surgen de la dificultad intrínseca de las estructuras de la L2, a menos que opten por evitar esas estructuras, como sería el caso de los hablantes de alemán e inglés en comparación con los francófonos en nuestro estudio. Por otra parte, evitar ciertas estructuras también puede deberse a la inesperada similitud entre la L1 y la L2 (Callies 2006), como lo verifica la tendencia entre hablantes de alemán que aprenden inglés a evitar verbos en posición final en inglés, estructura que es endémica en su L1, y a favorecer, en cambio, el patrón SVO que es prototípico del inglés. No obstante, en nuestro estudio se experimenta mayor interferencia cuando existen similitudes entre estructuras equivalentes en dos lenguas en estrecha relación (español-francés) que en el caso en que éstas son diferentes. Las equivalencias no son exactas, pero los sujetos parecen suponer una congruencia mayor que la real y transfieren patrones a formas y usos que de hecho no son paralelos, como lo demuestra el alto grado de transferencia en el caso de las oraciones subordinadas temporales del francés al español. Adicionalmente, están las diferencias individuales, de las que da cuenta debidamente Zybert (2007) y los factores sociolingüísticos presentados por Tarone (1982) y Odlin (1990), quienes indican que los estudiantes tienden a producir una mayor incidencia de formas cuidadas de la L2 en el habla monitoreada, más que en producciones espontáneas de estilo vernáculo, aduciendo que prestan más atención a la forma en que hablan y que en ambientes de sala de clases, donde la preocupación es mantener el buen nivel de la lengua, los interlocutores usan mayor precisión gramatical.

Finalmente, a la luz de descubrimientos recientes que provienen desde los afluentes de la lingüística cognitiva y la psicolingüística (Emmorey, 2003; Fabbro, 2002; Franceschini, Zappatore y Nitsch, 2003; Greene, 2005; Paradis, 2004; Ullman, 2006; Jarvis y Pavlenko, 2008), que indican que la transferencia también se produce desde la L2 a la L1 e incluso desde una L2 hacia una L3, concluimos que el fenómeno no es sólo un mecanismo de apoyo en la L1 cuando se carece de información sobre la L2, sino que la situación está conformada por una compleja interacción entre influencia interlingüística y principios naturales de la ASL que nos obligan a revisar nuestros conocimientos sobre la transferencia en la adquisición de segundas lenguas. 


\section{Referencias bibliográficas}

Biber, D., M. Davies, J. K. Jones, and N. Tracy-Ventura. (2006). “Spoken and written register variation in Spanish: A multidimensional analysis". Corpora. 1. 7-38.

Collentine, J. (2003). "The development of subjunctive and complexsyntactic abilities among FL Spanish learners". B. Lafford \& R. Salaberry (Eds.). Studies in Spanish second language acquisition: the state of the science. 74-97. Georgetown: Georgetown University Press.

Collentine, J. (2010). "The Acquisition and Teaching of the Spanish Subjunctive: An Update on Current Findings". Hispania. 93.1. 39-51.

Consejo de Europa (2002). Marco Común Europeo de Referencia para las Lenguas: Aprendizaje, Enseñanza, Evaluación. Instituto Cervantes para la Traducción en Español. $<$ http:/ / cvc.cervantes.es/ensenanza/ biblioteca_ ele/marco/cvc_mer.pdf $>$ Fecha de recuperación: 8 de julio de 2013.

Cook, V. (2003). Effects of the second language on the first. Clevedon, UK: Multilingual Matters.

Corder, S. (1978). "Language-learner language". En Jack, C. (Ed.). Understanding Second and Foreign Language Learning. 71-92. Rowley, Mass.: Newbury House.

Corder, S. P. (1983). "Strategies of Communication". C. Faerch \& G. Kasper (Eds.). Strategies in interlanguage communication. 15-19. Londres: Longman.

Chomsky, N. (1995). The Minimalist Program. Cambridge, Mass.: MIT Press.

Dechert, H. W. (2006). "On the ambiguity of the notion 'transfer'". En Cross-linguistic influences in the second language lexicon. Janusz Arabski (Ed.). 3-11. Clevedon, UK: Multilingual Matters. 
Doughty, C., Long, M. (2003). The handbook of second language acquisition. Wiley-Blackwell.

Eckman, F. (1984). "Markedness and the Contrastive Analysis Hypothesis". Language Learning. 27. 315-330.

Ellis, R. (1994). The Study of Second Language Acquisition. Oxford University Press.

Farley, A. (2004). "The Relative Effects of Processing Instruction and Meaning-Based Output Instruction". B. VanPatten (Ed.). Processing Instruction: Theory, Research, and Commentary. 143-168. Mahwah: Erlbaum.

Greenberg, J. (1966). "Some Universals of Grammar with Particular Reference to the Order of Meaningful Elements". Universals of Language. 73-113. Cambridge, Massachusets, and London, England: MIT Press.

Isabelli, C., Nishida, C. (2005). "Development of the Spanish Subjunctive in a Nine-Month Study-Abroad Setting". En Eddington, D. (Ed.). Selected Proceedings of the 6th Conference on the Acquisition of Spanish and Portuguese as First and Second Languages. Somerville, MA: Cascadilla. 78-91.

Jarvis, S., Pavlenko, A. (2008). Crosslinguistic influence in language and cognition. New York, London: Routledge.

Kellerman, E. (1987). Aspects of transferability in second language acquisition. Doctoral dissertation, Katholieke Universiteit te Nijmegen.

Krashen, S. (1985). The Input Hypothesis: Issues and implication. London, New York: Longman.

Meisel, J. M. (1980). “A Socio-psychological Approach to Natural Second Language Acquisition". En W. Hüllen (Ed.). Understanding Bilingualism. 83-113. Frankfurt.

Montrul, S. (2008). "Incomplete acquisition in Spanish heritage speakers: Chronological age or interface vulnerability?" Proceedings of the 32nd Annual Boston University Conference on Language Development. 299-310. Somerville, MA: Cascadilla Press. 
Newmark, L. y Reibel, D. A. (1968). "Necessity and sufficiency in language learning". IRAL 6.3.145-164.

Odlin, T. (2007). "Review of The Evolution of Language out of PreLanguage". Givón, T., Malle, B. (Eds.). Studies in Second Language Acquisition. 127.

Palmer, F. R. (2001). Mood and Modality. New York: Cambridge.

Paradis, M. (2004). A neurolinguistic theory of bilingualism. Amsterdam: John Benjamins.

Pienemann, M. (2011). “L1 transfer". Pienemann, M., J. Keßler. Studying Processability Theory. 75-83. Amsterdam: Benjamins.

Schachter, J. (1992). "A new account of language transfer". En S. Gass y L. Selinker (Eds.). Language transfer in language learning. 32-46. Amsterdam: John Benjamins.

Sorace, A. (2000). Differential effects of attrition in the L1 syntax of L2 nearnative speakers. Somerville, MA: Cascadilla Press.

Swan, M., Smith, B. (2001). Learner English: A Teacher's Guide to Interference and other Problems. Cambridge: Cambridge University Press.

VanPatten, B. (1997). “The Relevance of Input Processing to Second Language Theory and Second Language Teaching". Contemporary Perspectives on the Acquisition of Spanish. William Glass and Ana Pérez-Leroux (Eds.). 2. 93-108. Somerville: Cascadilla.

- y J. Williams, (2007). Theories in Second Language Acquisition: An Introduction. Mahwah, NJ: Erlbaum.

White, L. (1990). "Second language acquisition and universal grammar". Studies in Second Language Acquisition 12. 121-133. 